\title{
A Decomposition of Square Integrable Martingales
}

\author{
D. Isaacson
}

Z. Wahrscheinlichkeitstheorie verw. Gebiete 23, 327-330 (1972)

In [1] it is correctly pointed out that the proof of the theorem in my paper contains an unproved assertion. In particular it is not obvious that a process $\bar{N}_{s}$ with the stated properties will exist. Actually the process $\bar{N}_{s}$ is not needed in the proof so the theorem follows using the original process $N_{s}$ in the definition of $N_{t}^{*}$.

1. Mathematical Reviews 47, 1358 (1974)

\author{
Dean Isaacson \\ Statistical Laboratory and \\ Department of Statistics \\ Snedecor Hall \\ Ames, Iowa 50010, USA
}

